open revascularisation remained fairly constant. For men aged $<60$ years, the age-specific rate of amputations increased significantly $(\mathrm{p}=0.003)$ from 21.1 to 61.1 per 100000 over the study period $(\mathrm{R} R=1.33,95 \% \mathrm{CI} 1.09$ to 1.61). Therapeutic endovascular procedure rates increased significantly $(\mathrm{p}<0.001)$ for men aged 60 to 74 years $(\mathrm{RR}=1.65 ; 95 \% \mathrm{CI} 1.42$ to 1.92$)$ and 75 years or older $(\mathrm{RR}=2.6 ; 95 \%$ CI 1.96 to 3.47). Equally, a significant increase $(p<0.001)$ in the population rates of therapeutic endovascular procedures for women of all age groups occurred over the period examined.

Conclusions A substantial change in the practice of vascular surgery has occurred in Scotland in the last 2 decades perhaps in response to new technologies and new clinical guidelines. This change should inspire further research to determine the outcomes of these vascular procedures

\section{P1-454 EXPLANATIONS FOR SOCIAL INEQUALITIES IN PRETERM DELIVERY IN THE LIFEWAYS COHORT}

doi:10.1136/jech.2011.142976g.44

1,2 I Niedhammer, ${ }^{1} \mathrm{C}$ Murrin, ${ }^{1} \mathrm{D}$ O'Mahony, ${ }^{3} \mathrm{~S}$ Daly, ${ }^{4} \mathrm{~J}$ Morrison, ${ }^{1} \mathrm{C}$ Kelleher. ${ }^{*}$ UCD School of Public Health, University College Dublin, Dublin, Ireland; ${ }^{2}$ INSERM, U1018, CESP Centre for research in Epidemiology and Population Health, Epidemiology of occupational and social determinants of health Team, Villejuif, France; ${ }^{3}$ Coombe Women and Infant's University Hospital, Dublin, Ireland; ${ }^{4}$ National University of Galway, Galway, Ireland

Introduction Social inequalities in pregnancy outcomes have been extensively described, but studies that explain these inequalities comprehensively are lacking. This analysis evaluated the contribution of material, psychosocial, behavioural, nutritional, and obstetrical factors in explaining social inequalities in preterm delivery.

Methods The data were based on a prospective cohort of 1109 Irish pregnant women. Preterm delivery was obtained from clinical hospital records. Socio-economic status was measured using educational level. The association between educational level and preterm delivery was examined using Cox model.

Results Educational level was found to be a significant predictive factor of preterm delivery; women with low educational level were more likely to have a preterm delivery $(\mathrm{HR}=2.14,95 \% \mathrm{CI} 1.04$ to 4.38) after adjustment for age and parity. Rented and crowded home, smoking, alcohol consumption, and intake of saturated fatty acids displayed educational differences and were predictive of preterm delivery. Material factors (rented and crowded home) reduced the HR of preterm delivery for low educated women by $33 \%$. The independent contribution of behavioural factors (smoking and alcohol consumption) from material factors was $5 \%$, and the independent contribution of saturated fatty acids from material to behavioural factors was $4 \%$. All these factors together reduced the HR of preterm delivery for low educated women by $42 \%$ ( $\mathrm{HR}=1.66,95 \%$ CI 0.76 to 3.63$)$.

Conclusion This study is one of the first to attempt to explain social inequalities in preterm delivery comprehensively, and underlines the importance of material, behavioural and nutritional factors. More research is needed to better understand and prevent social inequalities in preterm delivery.

\section{P1-455 TOOTH LOSS ASSOCIATED WITH RACIAL/ETHNIC DISPARITIES: A STUDY PRO-HEALTH}

doi:10.1136/jech.2011.142976g.45

L Gonçalves, R Keller, ${ }^{*}$ J Bastos, E Faerstein. Federal University of Rio Grande do Sul, Porto Alegre, RS, Brazil

Tooth loss is the accumulation of oral health hazards such as lack of access to dental care, inappropriate health behavioural, low socioeconomic status. Studies have also shown more tooth loss among racial/ethnic minorities. Racial discrimination has been associated with racial/ethnic disparities in health, affecting the individual and population health. The study aims to evaluate the association between race-ethnicity and tooth loss and the role of socioeconomic status, health behaviours, health services access and self-reported discrimination. Baseline cross-sectional data were obtained from the Pro-Saúde Cohort Study (Rio de Janeiro-Brazil) in 4030 civil servants, and analysed with ordered logistic regression. The outcome was selfreported tooth loss measured in four ordered categories. In the unadjusted model, browns, blacks and other ethnic groups increased the chances of having more missing teeth if compared to white, respectively the OR was $2.46(p<0.01), 3.21(p<0.01)$ and 2.99 $(p=0.01)$. In the full model, adjusted for behavioural, socioeconomic, dental care and demographic variables, the OR was, respectively 1.27 $(p<0.05), 1.43(p<0.05)$ and $3.92(p<0.05)$ for browns, blacks and other ethnic groups respectively. There was no significant association between tooth loss and self-reported discrimination.

\section{P1-456 A CASE-CONTROL STUDY TO DETECT GENETIC AND ACQUIRED RISK FACTORS FOR PAEDIATRIC INFLAMMATORY BOWEL DISEASE}

doi:10.1136/jech.2011.142976g.46

${ }^{1} \mathrm{G}$ Kobashi, ${ }^{*} \mathrm{~A}$ Hata, ${ }^{1} \mathrm{~K}$ Ohta, ${ }^{3} \mathrm{H}$ Sugimori, ${ }^{4} \mathrm{~K}$ Okamoto, ${ }^{5} \mathrm{~A}$ Maekawa. ${ }^{1}$ National institute of radiological sciences, Chiba, Japan; ${ }^{2}$ Chiba University, Chiba, Japan; ${ }^{3}$ Daito Bunka University, Higashi matsuyama, Japan; ${ }^{4}$ Aichi Prefectural College of Nursing \& Health, Nagoya, Japan; ${ }^{5}$ Nagoya University, Nagoya, Japan

Intoduction Paediatric inflammatory bowel disease (PIBD) is considered to be a multifactorial disease with both genetic and acquired factors involved in its aetiology. The acquired factors include lifestyles and environmental factors of both patients in paediatric period and their mothers in perinatal period. Parental smoking, not breastfeeding (human milk substitute), mental stress, lack of sleeping time, low body activity, appendectomy, preterm delivery, and some genetic variants concerning pathways of immune responses such as CARD15/NOD2, DLG5, TLR4, OCTN1/2, MYO9B, IL23R, ATG16L, have reported to be possible risk factors for PIBD. However, to date, there has been no study analysing these factors simultaneously and clarifying their confoundings. The present study tries to elucidate genetic and acquired risk factors for PIBD and their confounding.

Methods PIBD cases and controls were recruited from affiliated hospitals of the Japan workshop for paediatric inflammatory bowel disease. Saliva sample of patients for genotyping and self-administrated questionnaire for their mothers were obtained with written informed-consent.

Result and conclusion This paper will report interim results of the study starting from 2011. The present study is expected to develop early and individualised measures to prevent PIBD, intervention for lifestyles and environmental factors of expectant mothers possessing genetic risk factors for baby's future PIBD manifestation. Further, the results may contribute to clarify new pathogenesis of PIBD manifestation and more useful disease classification.

\section{P2-457 WITHDRAWN}

\section{P1-458 TRENDS IN GEOGRAPHIC AND SOCIOECONOMIC DISPARITIES IN MUNICIPAL LIFE EXPECTANCY IN JAPAN: 1985-2005}

doi:10.1136/jech.2011.142976g.47

${ }^{1} \mathrm{~N}$ Kondo, ${ }^{*}{ }^{3} \mathrm{~K}$ Wada, ${ }^{2} \mathrm{~N}$ Ikeda, ${ }^{2} \mathrm{~K}$ Shibuya, ${ }^{2} \mathrm{E}$ Saito, ${ }^{1} \mathrm{Z}$ Yamagata. ${ }^{1}$ University of Yamanashi, Chuo-shi, Yamanashi, Japan; ${ }^{2}$ University of Tokyo, Bunkyo-ku, Tokyo, Japan; ${ }^{3}$ Kitasato University, Sagamihara-shi, Kanagawa, Japan

Introduction There is growing social concern about the expanding socioeconomic and health disparities that have occurred in Japan in 